$$
\text { CONF- } 9609280--3
$$

\title{
HARDNESS ENHANCEMENT AND CROSSLINKING MECHANISMS IN POLYSTYRENE IRRADIATED WITH HIGH ENERGY ION-BEAMS
}

\author{
EAL H. LEE, GOPAL R. RAO, and LOUIS K. MANSUR \\ Oak Ridge National Laboratory, P.O. Box 2008, Oak Ridge, TN 37831-6376
}

Kevwords: ion-beams, surface modification, radiation effects, crosslinking, scission, LET effects, hardness

\begin{abstract}
In the past, radiation effects studies on polymers were mostly aimed at understanding polymerization mechanisms in radiation curing and synthesis, and radiation induced degradation mechanisms. However, little effort has been expended to improve mechanical properties of polymers, because radiation sources such as e-beams, $\gamma$-rays, and ultraviolet light, which have been used frequently, produce either small improvement due to crosslinking or more often cause degradation due to scission. In recent work at ORNL, surface hardness values several times larger than that of steel were produced by employing high energy ion-beams (HEIB) in the range of several hundred keV to $\mathrm{MeV}$. Studies show that high linear energy transfer (LET) is important for crosslinking. At low LET conditions, induced active free radicals along the track are so sparsely dispersed that little interaction occurs among radicals. Input energy tends to localize at an intra-molecular chain segment, leading to chain scission. On the other hand, at high LET condition, massive ionization induces a high concentration of free radicals over many neighboring molecular chains facilitating crosslinking. Detailed crosslinking mechanisms are studied by analyzing hardness variations in response to irradiation parameters such as ion species, energy, and fluence. Effective crosslinking radii at hardness saturation are derived based on experimental data for $350 \mathrm{keV} \mathrm{H}^{+}$and $1 \mathrm{MeV} \mathrm{Ar}$ irradiation of polystyrene.
\end{abstract}

Research sponsored by the Division of Materials Sciences, U.S. Department of Energy, under contract No. DE-AC05960R22464 with Lockheed Martin Energy Research Corporation.
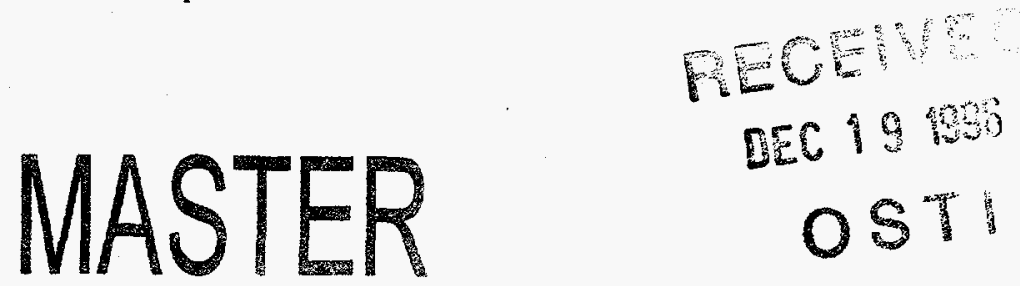

\section{DISTRIBUTION OF THIS DOCUMENT IS UNLAMTED}

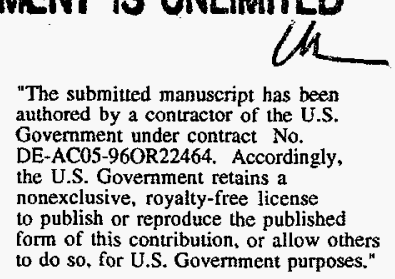




\section{DISCLATMER}

This report was prepared as an account of work sponsored by an agency of the United States Government. Neither the United States Government nor any agency thereof, nor any of their employees, makes any warranty, express or implied, or assumes any legal liability or responsibility for the accuracy, completeness, or usefulness of any information, apparatus, product, or process disclosed, or represents that its use would not infringe privately owned rights. Reference herein to any specific commercial product, process, or service by trade name, trademark, manufacturer, or otherwise does not necessarily constitute or imply its endorsement, recommendation, or favoring by the United States Government or any agency thereof. The views and opinions of authors expressed herein do not necessarily state or reflect those of the United States Government or any agency thereof. 


\section{DISCLAMMER}

Portions of this document may be illegible in electronic image products. Images are produced from the best available original document. 


\title{
HARDNESS ENHANCEMENT AND CROSSLINKING MECHANISMS IN POLYSTYRENE IRRADIATED WITH HIGH ENERGY ION-BEAMS
}

\author{
EAL H. LEE, GOPAL R. RAO, and LOUIS K. MANSUR \\ Oak Ridge National Laboratory, P.O. Box 2008, Oak Ridge, TN 37831-6376
}

Keywords: ion-beam, surface modification, radiation effects, crosslinking, scission, LET effects, hardness

\begin{abstract}
In the past, radiation effects studies on polymers were mostly aimed at understanding polymerization mechanisms in radiation curing and synthesis, and radiation induced degradation mechanisms. However, little effort has been expended to improve mechanical properties of polymers, because radiation sources such as e-beams, $\gamma$-rays, and ultraviolet light, which have been used frequently, produce either small improvements due to crosslinking or more often cause degradation due to scission. In recent work at ORNL, surface hardness values several times larger than that of steel were produced by employing high energy ion-beams (HEIB) in the range of several hundred $\mathrm{keV}$ to $\mathrm{MeV}$. Studies show that high linear energy transfer (LET) is important for crosslinking. At low LET conditions, induced active free radicals along the track are so sparsely dispersed that little interaction occurs among radicals. Input energy tends to localize at an intra-molecular chain segment, leading to chain scission. On the other hand, at high LET condition, massive ionization induces a high concentration of free radicals over many neighboring molecular chains facilitating crosslinking. Detailed crosslinking mechanisms are studied by analyzing hardness variations in response to irradiation parameters such as ion species, energy, and fluence. Effective crosslinking radii at hardness saturation are derived based on experimental data for $350 \mathrm{keV} \mathrm{H}^{+}$and $1 \mathrm{MeV} \mathrm{Ar}+$ irradiation of polystyrene.
\end{abstract}

\section{INTRODUCTION}

Organic polymers are inherently soft due to the lack of chemical bonds between polymer chains. When polymeric materials are subjected to high energy ion-beams (HEIB), however, a high number density of crosslinks are introduced, as well as numerous double and triple bonds, and free radicals. As a consequence, HEIB processed polymers become hard, electrically conductive, and optically dense, with improved resistance to chemicals and mechanical wear [1-5]. Hardness often improved over 50 times, exceeding the hardness values of steels. The property variations were analogous to each other because the improvements originated from the same cause, namely crosslinks. Hardness measurements have been used to investigate the materials' response to HEIB, since elastic properties and hardness are related to crosslink number density [6] and can be measured accurately and conveniently [7-9]. In this paper, therefore, ion-beam-polymer interaction mechanisms are investigated by scrutinizing the hardness variations in response to HEIB processing parameters, namely ion species, energy, and fluence. Since other property changes mentioned above were reported previously in references [1-5], they are not discussed in this paper. 


\section{Mechanisms}

When an energetic particle impinges into a polymer medium with high velocity, its orbital electrons are stripped to varying degrees depending upon the velocity of the particle. The maximum achievable charge state of the particle depends upon the number of protons. The velocity required to achieve the highest ionized state also depends upon the atomic number. The higher the number of protons the particle has, the higher would be the coulombic force exerted when orbital electrons are stripped off, but the higher mass ion would also require higher energy to be accelerated to a given velocity. The stripped ion eventually reacquires its orbital electrons as it slows down below the velocity of the Bohr electron [10]. When a charged particle passes through a medium, it sets nearby electrons in motion by its electric field and also creates a large number of secondary electrons by knock-on collisions. The particle thus looses its energy by transferring its energy to the medium until it slows down and stops. These energy loss processes are known as 'electronic stopping.' The particle also looses its energy by displacing atoms in the medium by nuclear collisions. These energy loss processes are known as 'nuclear stopping.' While most electronic energy loss stems from primary ions, most nuclear energy loss comes from recoil atoms caused by primary ions, because many recoil atoms can be created by a primary ion and they have lower energies and thus have larger nuclear stopping cross-section. Although recoil atoms also loose their energies by electronic processes, their contributions are small compared to those of primary ions because low energy recoil atoms have small cross section for electronic stopping. For small atoms such as $\mathrm{H}$ or $\mathrm{He}$, nuclear stopping is negligible because their nuclear collisional cross-sections are very small at most energies of interest. 'Nuclear stopping,' however, becomes important for ion species with a larger number of nucleons. The unit $\mathrm{eV} / \mathrm{nm} / \mathrm{ion}$ or simply $\mathrm{eV} / \mathrm{nm}$ is used for the energy loss per unit path length or linear energy transfer (LET).

Finally, excited lattice atoms and electrons lose their energy through vibrational decay as phonons and plasmons, respectively. Phonons and plasmons decay mostly through thermal and radiative processes. Although energy loss by phonon decay becomes significant for large atoms, no obvious thermal effect was observed within the range of irradiation conditions employed in this work. Energy loss by plasmon decay is very small and its contribution would be negligible even if it affects material properties. Experimental evidence showed that modification of polymer properties depended mainly upon electronic $\mathrm{LET}_{e}$ and nuclear $\mathrm{LET}_{n}[2]$.

During irradiation, various physical and chemical processes take place in the polymer. Coulombic interactions between ions and electrons of host atoms, excessive bond stretching due to localized energy deposition, and atomic displacement by nuclear collision can release pendant atoms such as hydrogen, and cause bond breakage or chain scission. Thus, various gaseous molecular species are released during irradiation. The most prominent species are hydrogen, molecular scission products from the end groups as well as pendant groups of the polymer, and their reaction products. Radicals or dangling bonds are created by releasing pendant atoms such as hydrogen. Crosslinking occurs if a union of two free dangling bonds occurs between the neighboring chains, and formation of double or triple bonds occurs if two neighboring radicals in the same chain unite.

It has been well established that mechanical, physical, and chemical property changes in polymers are determined by the magnitude of crosslinking and scission, and that crosslinking enhances 
mechanical stability while scission degrades mechanical strength [11]. Although both electronic and nuclear energy transfer can induce crosslinking as well as scission, as would be intuitively expected, experimental evidence suggests that electronic stopping causes more crosslinking while nuclear stopping causes more scissions $[2,12]$. This provides much of the basis for understanding the materials' response to irradiation.

\section{EXPERIMENTAL}

Polystyrene (PS) sheets used in this experiment were purchased from Goodfellow Advanced Materials Company, Malvern, PA. The polymer was separately irradiated with $350 \mathrm{keV} \mathrm{He}^{+}$and $1 \mathrm{MeV} \mathrm{Ar}{ }^{+}$ions at the Triple Ion-beam Irradiation Facility in the Metals and Ceramics Division at ORNL. Details about the facility can be found in reference [13]. Beam currents under $1 \mathrm{~mA} \mathrm{~m}^{-2}$ were used, maintaining the temperature of the polymer surface below $100^{\circ} \mathrm{C}$ to avoid overheating and degradation of the polymer surface. Surface hardness values were measured at $100 \mathrm{~nm}$ depth with the Nanoindenter ${ }^{\otimes}$. Details of hardness measurement procedures can be found in reference [14].

\section{RESULTS}

In Fig. 1(a), surface hardness values of polystyrene irradiated with $350 \mathrm{keV} \mathrm{He}^{+}$and $1 \mathrm{MeV} \mathrm{Ar}^{+}$ ions are plotted as a function of fluence (number of ions injected per unit area of target material, ions $\mathrm{m}^{-2}$ ). Although comparing the hardness values at an equivalent fluence is useful when one is concerned with the number of ions required to cover the subject target area, the effect of energy deposition or LET cannot be clearly delineated because the energies deposited by the $350 \mathrm{keV}$ $\mathrm{He}^{+}$and $1 \mathrm{MeV} \mathrm{Ar}{ }^{+}$are different at the same fluence. Therefore, hardness values are replotted as a function of dose (energy deposition per unit mass of target material) in Fig. l(b). Here, the SI unit of Gray (Gy) is obtained by multiplying the fluence (ions $\mathrm{m}^{-2}$ ) with LET (eV/nm/ion) at the surface and dividing by the specific gravity of polystyrene $\left(1.05 \times 10^{3} \mathrm{~kg} / \mathrm{m}^{3}\right)$ and finally using a conversion factor of $1 \mathrm{~Gy}=6.24 \times 10^{18} \mathrm{eV} / \mathrm{kg}$.

The results showed that surface hardness increased with fluence and reached a saturation value of $\sim 20 \mathrm{GPa}$ near a fluence of $2 \times 10^{20} \mathrm{~m}^{-2}$ for $1 \mathrm{MeV} \mathrm{Ar}$, and near $7 \times 10^{20} \mathrm{~m}^{-2}$ for $350 \mathrm{keV} \mathrm{He}^{+}$as indicated by dotted vertical lines in Fig. 1(a). When plotted as a function of dose, however, the trends of hardness value appeared to saturate at the same dose of $\sim 3 \times 10^{10} \mathrm{~Gy}$ for both $\mathrm{He}^{+}$and $\mathrm{Ar}^{+}$ions as indicated by a dotted vertical line in Fig. 1(b). However, hardness values for $350 \mathrm{keV}$ $\mathrm{He}^{+}$were lower at all doses than those for $1 \mathrm{MeV} \mathrm{Ar}^{+}$until hardness saturated. When the hardness data were extrapolated to the $x$-axis as indicated by the solid lines in Fig. 1(b), apparent incubation doses (here it is defined to be a dose required to improve hardness to $10 \%$ above the pristine unirradiated value) were found to be $\sim 2 \times 10^{8} \mathrm{~Gy}$ for $350 \mathrm{keV} \mathrm{He}{ }^{+}$, and $\sim 1 \times 10^{7}$ Gy for 1 $\mathrm{MeV} \mathrm{Ar}$ indicating that $\mathrm{He}^{+}$required about 20 times higher incubation dose.

\section{DISCUSSION}

The hardness data presented above are examined in terms of three critical parameters, ion species, ion energy, and fluence.

In Fig. 2, the electronic and nuclear energy loss profiles for $350 \mathrm{keV} \mathrm{He}{ }^{+}$and $1 \mathrm{MeV} \mathrm{Ar}^{+}$are plotted to examine the effects of ion species and energy using the Monte Carlo simulation code 
TRansport of Ions in Matter (TRIM) [10]. All energy loss calculations in the following section are made using the PC-TRIM code (version 95.06).

Electronic $\mathrm{LET}_{\varepsilon}$ values for $350 \mathrm{keV} \mathrm{He}{ }^{+}$and $1 \mathrm{MeV} \mathrm{Ar}{ }^{+}$are about 260 and $960 \mathrm{eV} / \mathrm{nm}$ at the surface, respectively. Most nuclear energy loss occurs near the end of the ion track where recoil atoms are prevalent and have low energies. At high energies, nuclear cross-section is small and only few of the many encounters in the target cause significant deflection from the straight path of flight. Since the nuclear energy loss at the surface region is small compared to the electronic energy loss, less than a few percent for $\mathrm{Ar}$ and almost negligible for $\mathrm{He}$, the hardness changes at the surface region can be considered mostly due to the electronic energy loss.

It is interesting to note that the surface $\mathrm{LET}_{\mathrm{e}}$ ratio for $\mathrm{He}^{+}$to $\mathrm{Ar}^{+}$is about $260(\mathrm{He}) / 960(\mathrm{Ar}) \approx$ $1 / 3.7$, which is approximately equal to the inverse of the saturation fluence ratio, $7 \times 10^{20}$ $(\mathrm{He}) / 2 \times 10^{20}(\mathrm{Ar}) \approx 3.5$. This result suggests that the higher the LET, the lower would be the fluence required to achieve a saturation hardness such that total deposition energy per unit mass of target would be about equal at saturation. This point is further verified in Fig. l(b), which shows that the trends of hardness value appear to saturate at the same dose near $\sim 3 \times 10^{10} \mathrm{~Gy}$ for both $\mathrm{He}^{+}$and $\mathrm{Ar}^{+}$.

An important question then is 'do we need high LET to achieve a desired hardness?' We have seen above that $350 \mathrm{keV} \mathrm{He}{ }^{+}$would require 3.5 times higher fluence than $1 \mathrm{MeV} \mathrm{Ar}{ }^{+}$at the same ion flux to attain the saturation hardness. Irradiation with $1 \mathrm{MeV}$ electrons $\left(\mathrm{LET}_{\mathrm{c}} \approx 0.19 \mathrm{eV} / \mathrm{nm}\right.$ ) would require $10^{4}$ times higher fluence if the same assumption of linearity applies. For this reason, although radiation sources such as electron beams and $\gamma$-rays have been employed successfully in areas where moderate crosslinking is sufficient for applications as in radiation curing and photolithography, a large improvement in hardness was not generally observed in these cases. However, as will be discussed further below, for low LET, radical pairs or dangling bonds are produced so sparsely that the probability of two neighboring radicals to be close enough for crosslinking is very low. In such cases, a significant fraction of deposited energy remains within chains, leading frequently to chain scission and thus degradation of material.

Schnabel et al. [15] observed an increasing trend of scission with decreasing LET for PMMA irradiated by various high energy irradiation sources as summarized in Table 1. In their experiment, $G(s)$ values for random main-chain scission were determined from molecular weight distribution via gel permeation chromatography. G-value is a quantitative measure of the specific chemical yield (here scission) per $100 \mathrm{eV}$ of radiation energy absorption. In consistency with the reasons mentioned above, $\gamma$-rays produced the highest scission because of low LET despite the purely ionizing nature of the radiation (no nuclear displacements). 
Table 1 - G(s) values for scission in irradiated PMMA [15]

\begin{tabular}{|c|c|c|}
\hline Ion Energy & LET (eV/nm) & $\mathbf{G}(\mathbf{s})$ \\
\hline${ }^{60} \mathrm{Co} \gamma$-rays & 0.2 & 1.5 \\
$45 \mathrm{MeV} \mathrm{He}^{2+}$ & 20 & 0.8 \\
$240 \mathrm{MeV} \mathrm{Ne}^{7+}$ & 476 & 0.3 \\
$90 \mathrm{MeV} \mathrm{O}^{4+}$ & 577 & 0.3 \\
\hline
\end{tabular}

A second question then is 'why do $350 \mathrm{keV} \mathrm{He}{ }^{+}$and $1 \mathrm{MeV} \mathrm{Ar}$ give the same saturation hardness despite the difference in LET?' To answer this question, let us examine PMMA example again. PMMA is a scissioning type polymer [16]. When PMMA was subjected to low LET beam sources such as $1.5 \mathrm{MeV} \mathrm{H}^{+}, 1 \mathrm{MeV} \mathrm{He}{ }^{+}$, and $20 \mathrm{keV}$ electron beam, initially it became more soluble in a solvent at low fluences but eventually became insoluble at high fluences [17]. The reason for this peculiar solubility behavior of PMMA is illustrated schematically in Fig. 3. For scissioning type polymers, at low fluences, average molecular chain length decreases because of predominant scission. At high fluences, however, scissioned segments are connected by random crosslinks making it difficult to dissolve. For this reason, at low LET, the crosslinked region may not have the same degree of mechanical integrity as for the high LET case, even if an apparent saturation hardness could be attained at high fluences. It should be noted that hardness of a polymer is affected by crosslink density as well as by chain mobility. Thus, a polymer can be hard even if numerous scissions occur in the chains when overall chains are locked up by crosslinks as shown in Fig. 3. Although PMMA degraded under low LET conditions, when PMMA was treated with high LET ions such as $2 \mathrm{MeV} \mathrm{Ar}\left(\mathrm{LET}_{e} \approx 1130 \mathrm{eV} / \mathrm{nm}\right)$, a smooth film with hardness of $10.8 \mathrm{GPa}$ was obtained at a fluence of $1 \times 10^{19}$ ions $\mathrm{m}^{-2}$ because of enhanced crosslinking [5]. There is evidence that, when crosslinkable sites are exhausted, such as at saturation, further irradiation may induce scissions $[4,18]$. In such a case, hardness reduction may not be noticeable until very severe degradation occurs because of restricted chain mobility. However, electrical conductivity, which is sensitive to carrier mobility or chain connectivity, decreased after a maximum conductivity was reached indicating that additional irradiation indeed degraded chain connectivity $[4,18]$.

In materials with low atomic numbers, such as organic polymers, the oscillator strength distribution is confined effectively to an excitation energy below $100 \mathrm{eV}$, and the average energy loss events involve an energy between 30 and $40 \mathrm{eV}[19,20]$. This track entity involving a small isolated energy deposit is called a spur. An average energy required to produce a pair of ions or a pair of radicals in polymers has also been found to be tantamount to spur energy [19-21]. For low LET irradiations, spurs are widely separated like a string of beads. For high LET, the spurs overlap and form a continuous column facilitating cross-linking. Assuming an average spur energy to be $35 \mathrm{eV}$, for $350 \mathrm{keV} \mathrm{He}{ }^{+}$with $260 \mathrm{eV} / \mathrm{nm}$ LET, the average distance between spurs is then about $35 / 260 \approx 0.13 \mathrm{~nm}$, larger than $\mathrm{C}-\mathrm{H}$ bond distance $(\sim 0.11 \mathrm{~nm})$. It would, therefore, be difficult to create two dangling (C- $-\mathrm{C}$ ) bonds nearby simultaneously and crosslink them together in a single ion track. On the other hand, for $1 \mathrm{MeV} \mathrm{Ar}{ }^{+}$, the average distance between spurs is 
$35 / 960 \approx 0.037 \mathrm{~nm}$, almost 3 times shorter than the $\mathrm{C}-\mathrm{H}$ bond distance $(0.11 / 0.037 \approx 3)$. In this case, spurs are so closely spaced that almost three spurs can overlap within a $\mathrm{C}-\mathrm{H}$ bond distance $(\sim 0.11 \mathrm{~nm})$.

It required many passes of ions, approximately $700 \mathrm{He}^{+}$ions $/ \mathrm{nm}^{2}$ or $200 \mathrm{Ar}^{+}$ions $/ \mathrm{nm}^{2}$ to attain the saturation hardness, see Fig. 1(a). Though it is unlikely, if there were no overlaps of ion tracks, effective radius of ion track at saturation can be calculated to be $0.02 \mathrm{~nm}$ and $0.04 \mathrm{~nm}$ for 350 $\mathrm{keV} \mathrm{He}{ }^{+}$and $1 \mathrm{MeV} \mathrm{Ar}^{+}$, respectively, using $n_{i} \pi r_{e}{ }^{2}=1 \mathrm{~nm}^{2}$ relationship where $n_{i}$ is the number of ions injected to $1 \mathrm{~nm}^{2}$ area at saturation. The reason for the small effective radius can be further elucidated if one considers the fact that polystyrene has 18 monomer units in a rhombohedral cell with lattice constants of $a_{0}=2.2 \mathrm{~nm}, b_{0}=2.2 \mathrm{~nm}$ and $c_{0}=0.663 \mathrm{~nm}[22,23]$. Although the polystyrene studied in this work is not a crystalline form, it is expected that the average distance between two polymer chains is larger than $1 \mathrm{~nm}$. In this situation, it would be very difficult for two radicals to approach to a cross-linking distance, say the $C-C$ bond distance $(\sim 0.15 \mathrm{~nm})$. Experimental evidence, however, suggested that crosslinking occurs after substantial emission of hydrogen atoms followed by material compaction or an increase in specific gravity. In our unreported experiment, measurements of specific gravity using a density gradient column showed that specific gravity of pristine polycarbonate was $1.2 \times 10^{3} \mathrm{~kg} / \mathrm{m}^{3}$ and that of the bombarded layer (extracted by dissolving away the unbombarded substrate) was $1.54 \times 10^{3} \mathrm{~kg} / \mathrm{m}^{3}$ after irradiating to a fluence of $1 \times 10^{19}$ ions $/ \mathrm{m}^{2}$ with $2 \mathrm{MeV} \mathrm{O}^{+}$ions. The result suggests that significant removal of hydrogen was required to eliminate enough space for polymer chain segments to move to a cross-linking distance. When the saturation dose $3 \times 10^{10}$ Gy was converted to $1.97 \times 10^{5} \mathrm{eV} / \mathrm{nm}^{3}$ and a $35 \mathrm{eV}$ per spur conversion factor was used, it turned out that almost 5600 spurs $\left(1.97 \times 10^{5} / 35 \approx 5600\right)$ were deposited per $\mathrm{nm}^{3}$ at saturation for both He and Ar. When one considers that there are about 6 monomer units per $\mathrm{nm}^{3}$ in polystyrene, every monomer unit encountered 933 spurs $(5600 / 6 \approx 933)$ at saturation. This result suggests that most crosslinking occurred in the region where ion tracks overlap rather than along a single ion track.

A third question is then 'why hardness values for $350 \mathrm{keV} \mathrm{He}^{+}$were lower at all doses than those for $1 \mathrm{MeV} \mathrm{Ar}^{+}$until hardness saturated?' and 'why apparent incubation dose for $350 \mathrm{keV} \mathrm{He}^{+}$ was higher than that for $1 \mathrm{MeV} \mathrm{Ar}^{+}$?', Fig. 1(b). As discussed above, the experimental results suggested that most crosslinking occurred in the area where ion tracks overlap. The answer can then be provided by demonstrating that an overlap of ion tracks should occur earlier for ions with larger effective crosslinking radii, or with higher spur density. In a stochastic process of ion track development, since an average distance between two ion track centers for the same fluence would be about equal regardless of track radii, the probability of track overlap increases with increasing track radii, as shown in Fig. 4. Actual crosslinking radius for $350 \mathrm{keV} \mathrm{He}^{+}$and $1 \mathrm{MeV} \mathrm{Ar}^{+}$could not be derived in this experiment. However, since the surface $\mathrm{LET}_{e}$ ratio for $\mathrm{He}^{+}$to $\mathrm{Ar}^{+}$is about $260(\mathrm{He}): 960(\mathrm{Ar}) \approx 1: 4$ and the ratio of effective radius square at saturation $\left(\mathrm{r}_{3}^{2}\right)$ was found to, be about $(0.02)^{2}(\mathrm{He}):(0.04)^{2}(\mathrm{Ar})=1: 4$, let's assume that the ratio of effective crosslinking radius for $\mathrm{He}^{+}$and $\mathrm{Ar}^{+}$is approximately $r_{e}(\mathrm{He}): r_{e}(\mathrm{Ar}) \approx 1: 2$ and that the energy density within the radius is uniform. Then, one can illustrate track overlap progress at three different equivalent doses (e.g., four He ion tracks to one Ar track) as shown in Fig. 5. The tracks have been forced to fill the unbombarded area first to visualize which size of ion tracks overlaps sooner. In the figure, single, double, and triple overlap regions are indicated with increasing shade density. Indeed, 
multiple track overlap occurs earlier for the high LET case as expected.

However, there are reports that the crosslinking $\mathrm{G}(\mathrm{x})$ value for polystyrene is invariant with LET for a wide range of LET values, contradicting the current observation. The final question is then how this disagreement arises. Before answering this question, let us review the reported data first. The $\mathrm{G}(\mathrm{x})$ values were found to be within a range of 0.03 to $0.05[15,24,25]$, when polystyrene was subjected to $1.25 \mathrm{MeV}$ (average) ${ }^{60} \mathrm{Co \gamma}$-rays $(0.2 \mathrm{eV} / \mathrm{nm}), 275 \mathrm{MeV} \mathrm{Ne}{ }^{7+}(370$ $\mathrm{eV} / \mathrm{nm})$, and $180 \mathrm{MeV} \mathrm{Ar}^{8+}(2260 \mathrm{eV} / \mathrm{nm})$ as summarized in Table II. On the other hand, Calcagno et. al [26] reported a $\mathrm{G}(\mathrm{x})$ value of 0.3 for $100 \mathrm{keV} \mathrm{He}{ }^{+}(210 \mathrm{eV} / \mathrm{nm}), 200 \mathrm{keV} \mathrm{Ne}^{+}$ $(450 \mathrm{eV} / \mathrm{nm})$, and $400 \mathrm{keV} \mathrm{Ar}{ }^{+}(850 \mathrm{eV} / \mathrm{nm})$ irradiated polystyrene showing an order of magnitude larger value than those reported previously. In their work, 200-300 nm thin spin cast films were used to ensure uniform energy deposition and to minimize nuclear contribution. The discrepancy between the data of Calcagno et. al and the previous data appears to originate perhaps from computational difference rather than actual difference in $G$ value because both data were derived from a similar range of LET. Calcagno et al. attributed their high $\mathrm{G}$ value to high LET by comparing with low LET $\gamma$-rays data without acknowledging the data of Schnabel et al. Nonetheless, if we consider the data of Calcagno et al. and previous data separately, all data can be interpreted consistently to indicate that the $G(x)$ value of polystyrene is almost invariant with LET. The reason for this is explained below.

Table II $G(x)$ values for polystyrene subjected to a wide range of LET irradiation sources

\begin{tabular}{|l|c|c|l|}
\hline \multicolumn{1}{|c|}{ Ion Energy } & LET $(\mathrm{eV} / \mathbf{n m})$ & $\mathbf{G}(\mathbf{x})$ & \multicolumn{1}{c|}{ References } \\
\hline${ }^{60} \mathrm{Co} \gamma$-rays & 0.2 & 0.05 & Burlant [24] \\
$1.25 \mathrm{MeV}$ avg. & & $0.03-0.05$ & Parkinson [25] \\
& 370 & 0.05 & Schnabel [15] \\
$275 \mathrm{MeV} \mathrm{Ne}^{7+}$ & 2260 & 0.03 & \\
$180 \mathrm{MeV} \mathrm{Ar}^{8+}$ & 210 & 0.3 & Calcagno [26] \\
\hline $100 \mathrm{keV} \mathrm{He}^{+}$ & 450 & 0.3 & \\
$200 \mathrm{keV} \mathrm{Ne}^{+}$ & 850 & 0.3 & \\
$400 \mathrm{keV} \mathrm{Ar}^{+}$ & & & \\
\hline
\end{tabular}

In most experiments, $\mathrm{G}(\mathrm{x})$ values were derived from the molecular weight distributions determined by gel permeation chromatography (GPC) $[15,26]$ or viscosity measurements $[24]$ after dissolving irradiated polymers in a solvent. This requires polymers to be still soluble after irradiation. Therefore, very low fluence of ions, generally much below $10^{16}$ ions $/ \mathrm{m}^{2}$ (or less than one ion per $1000 \mathrm{~nm}^{2}$ ), was applied to ensure solubility of the irradiated polymer. At this low fluence regime, ion tracks are so widely separated that most crosslinking may occur in a single ion track, probably in regions where two polymer chains are tangled or crossover each other such that they are within crosslinking distance. In such a case, higher LET ions would of course produce more crosslinks per ion but the number of crosslinks produced per unit deposited energy would be the same regardless of LET. Thus $G(x)$ value is independent of LET for low fluence or in the single ion track regime.

From the data shown in Fig. 1(b) and discussions presented above, it can be considered that there 
are three regimes of crosslinking based on fluence: 'low fluence or single ion track regime' where ion tracks are isolated and the number of crosslinks produced is proportional to the deposited energy only, but $\mathrm{G}(\mathrm{x})$ is invariant with LET; 'intermediate fluence or track overlap regime' where enhanced crosslinking occurs in track overlap regions and $G(x)$ varies with LET (track radius or spur density); and finally 'high fluence or saturation regime' where most crosslinkable sites are exhausted and further irradiation may induce more scission than crosslinking and degrade the material. Idealized these three regimes are illustrated in Figure 6 . In 'single ion track regime' $\mathrm{G}(\mathrm{x})$ is low. In 'track overlap regime' crosslinking is enhanced and thus $\mathrm{G}(\mathrm{x})$ is high. In saturation regime $G(x)$ would decline again though this may not be detected by hardness measurement. If we define the incubation period as an onset of track overlap, it can now be understood why the low LET $\mathrm{He}^{+}$showed a longer incubation period. This is because track overlap is delayed due to the smaller track radii.

It is, however, interesting to note that although incubation period or onset of track overlap is delayed for low LET, the higher slope of the curve in the track overlap regime suggests that crosslinking rate is higher once track overlap is initiated. In the discussion above in the demonstration of track overlap effect for low and high LET situation (Fig. 5), we assumed that the effective radius ratio was $r_{e}(\mathrm{He}): r_{e}(\mathrm{Ar}) \approx 1: 2$ based on the effective radius values derived at saturation if there were no track overlap and that energy density within the track radii is constant. However, in reality the chemical core radius (r) increases almost linearly with LET within the energy range of interest (e.g., up to $0.4 \mathrm{MeV}$ per nucleon for $\mathrm{He}$ and $1 \mathrm{MeV}$ per nucleon for $\mathrm{Ar}$ ) and decreases to a physical core radius with further increase of LET beyond these energies [see Fig. 6-1 in Ref. 19], and thus the real ratio of $r_{e}(\mathrm{He}): r_{e}(\mathrm{Ar})$ might have been smaller than 1:2, probably 1:4 if actual track radii could be measured, and track overlap for low LET might have been delayed even further. If the radius increases linearly with LET, energy density would decline more rapidly for high LET since the radial area increases as $r^{2}$. For higher LET (e.g., 1 $\mathrm{MeV} \mathrm{Ar}^{+}$), a total or an accumulated crosslinking yield, $\mathrm{G}_{1}(\mathrm{x})$ value, would be higher because of earlier track overlap, but an instantaneous crosslinking yield (slope of the curve) in the intermediate dose range, $G_{i}(x)$ value, would be lower compared to that of lower LET (e.g., 350 $\mathrm{keV} \mathrm{He}^{+}$) due to lower energy density in the track overlap regions, such that a saturation hardness occurs at a similar dose.

\section{Implications}

The degree of crosslinking and the depth of the ion-beam modified layer can now be controlled based on physical principles, namely by controlling the three major processing parameters, ion species, energy, and fluence. Although apparent saturation hardness was the same for both low and high LET, experimental evidence suggested that materials may degrade in a very low LET environment because of dominant scissions, as in weathering by UV radiation. Therefore, it is recommended that high energy ion-beams (HEIB) be used, when highly crosslinked and mechanically sound surfaces are desirable. Besides, low LET irradiations require longer processing times, making the process uneconomical. Finally, HEIB provides an unique opportunity for industrial applications in areas where light weight polymeric materials with high surface hardness, wear and scratch resistance, oxidation and chemical resistance, diffusion or permeation barrier, and improved electrical conductivity are required. Specific examples for the property improvements can be found in references [1-5]. 


\section{Conclusions}

Crosslinking mechanisms in polymeric materials were investigated by examining hardness variation of polystyrene subjected to $350 \mathrm{keV} \mathrm{He}^{+}$ions $\left(\mathrm{LET}_{\mathrm{e}} \approx 260 \mathrm{eV} / \mathrm{nm}\right.$ ) and $1 \mathrm{MeV} \mathrm{Ar}$ ions $\left(\mathrm{LET}_{\mathrm{e}} \approx 960 \mathrm{eV} / \mathrm{nm}\right)$. The results showed that hardness improvement was greater for higher LET ion species at equivalent fluences as well as at equivalent doses, indicating that high LET is more effective in producing crosslinks. However, the saturation hardness was attained at the same dose of $3 \times 10^{10}$ Gy regardless of LET, suggesting that dangling radicals are long lived and all possible crosslinking occurs eventually as new radicals are created in the vicinity. Analysis indicated that spurs are separated by $0.13 \mathrm{~nm}$ for $350 \mathrm{keV} \mathrm{He}{ }^{+}$and by $0.037 \mathrm{~nm}$ for $1 \mathrm{MeV} \mathrm{Ar}$. When spurs are widely spaced as for the He case, a larger incubation fluence was required before the onset of hardness enhancement. This was explained in terms of effective crosslinking radii. Crosslinking rate is low in single ion track regime and high in track overlap regime. Lower LET has smaller effective track radii, and thus track overlap or hardness enhancement is delayed. In single ion track regime, $G(x)$ is proportional to absolute deposit energy and is invariant with LET. In track overlap regime, crosslinking yield, $G(x)$, is enhanced because of track overlap. However, although the higher LET $\left(1 \mathrm{MeV} \mathrm{Ar}^{+}\right)$showed the higher total crosslinking yield, $\mathrm{G}_{\mathrm{t}}(\mathrm{x})$, because of earlier track overlap, the instantaneous $\mathrm{G}_{\mathrm{i}}(\mathrm{x})$ was lower compared to that of the lower LET $\left(350 \mathrm{keV} \mathrm{He}\right.$ ) once in the track overlap regime, because energy density decreases as $\mathrm{r}^{-2}$ with increasing track radius. At high fluences, when crosslinkable sites are exhausted, scission becomes dominant and the polymer degrades. Although the mechanisms described above are plausible, a detailed theoretical model should be developed in the future to better define and understand the track overlap mechanisms and the data.

\section{Acknowledgment}

This research was sponsored by the Division of Materials Sciences, U.S. Department of Energy, under contract No. DE-AC05-96OR22464 with Lockheed Martin Energy Research Corporation. The authors wishes to thank $\mathrm{Dr}$. M. B. Lewis and Dr. J. S. Lin for manuscript review and helpful discussions. 


\section{References}

[1] E. H. Lee, M. B. Lewis, P. J. Blau, and L. K. Mansur, J. Mater. Res., 6, 610 (1991).

[2] E. H. Lee, G. R. Rao, M. B. Lewis, and L. K. Mansur, J. Mater. Res., 9, 1043 (1994).

[3] G. R. Rao, E. H. Lee, R. Bhattacharya, and A. W. McCormick, J. Mater. Res. 1, 190 (1995).

[4] E. H. Lee, Chapter 17 in"Polyimide: Fundamental Aspects and Technological Applications," eds. K. Mittal and M. Ghosh, Marcel Dekker Inc., New York, 471 (1996).

[5] E. H. Lee, G. R. Rao, and L. K. Mansur, Trends in Polymer Science, 4, 229 (1996).

[6] A. Charlesby, Radiat. Phys. Chem., 40, 117 (1992).

[7] F. J. Baltá Calleja, Adv. Polym. Sci. 66, 117 (1985).

[8] E. H. Lee, Y. Lee, W. C. Oliver, and L. K. Mansur, J. Mater. Res. 8, 377 (1993).

[9] G. R. Rao, L. Riester, and E. H. Lee, Mat. Res. Soc. Symp. Proc., 345, 363 (1995).

[10] J. F. Ziegler, J. P Biersack, and U. Littmark, "The Stopping and Range of Ions in Solids," Vol. 1, (Pergamon Press, 1985).

[11] J. M. Cowie, in "Polymers: Chemistry \& Physics of Modern Materials," Intertext Books, Billings \& Son Ltd. Worcester, Great Britian, 283 (1973).

[12] M. B. Lewis, E. H. Lee, and G. R. Rao, J. Nucl. Mater. 211, 46 (1994).

[13] M. B. Lewis, W. R. Allen, R. A: Buhl, N. H. Packan, S. W. Cook, and L. K. Mansur, Nucl. Instrum. Methods B43, 243 (1989).

[14] E. H. Lee, Y. Lee, W. C. Oliver, and L. K. Mansur, J. Mater. Res. 8, 377 (1993).

[15] Wolfram Schnabel, Siegfried Klaumünzer, Hideto Sotobayashi, Frithjof Asmussen, and Yoneho Tabata, Macromolecules 17, 2108 (1984).

[16] A. Shapiro, in "Radiation Chemistry of Polymeric Systems," Interscience Publishers, John Wiley \& Sons, New York, 353 (1962)

[17] T. M. Hall, A. Wagner, and L. F. Thompson, J. Appl. Phys. 53, 3997 (1982).

[18] Y. Wang, S. S. Mohite, and L. B. Bridwell, J. Mater. Res. 8, 388 (1993).

[19] J. L. Magee and A. Chatterjee, "Radiation Chemistry, Principles and Applications,"

Farhataziz and Michael A. J. Rodgers, Eds., VCH Publishers, New York (1987).

[20] J. L. Magee and A. Chatterjee, "Kinetics of Nonhomogeneous Processes," Gordon R.

Freeman, Ed., John Wiley \& Sons, New York, 184 (1987).

[21] ICRU Report 31, "Average Energy Required to Produce An Ion Pair," International

Commission on Radiation Units and Measurements, Washington D.C. 1979.

[22] R. L. Miller and L. E. Nielsen, J. Polymer Science, 55, 643 (1961).

[23] Polymer Handbook, 3rd edition, J. Brandrup and E. H. Immergut, eds., John Wiley \& Sons, Inc., New York, (1989).

[24] W. Burlant and J. Neerman, J. Polymer Science, 58, 491 (1962).

[25] W. W. Parkinson and R. M. Keyser in "The Radiation Chemistry of Macromolecules," ed. M. Dole, Vo. II, p 72 (Academic Press, New York, 1973).

[26] L. Calcagno, G. Foti, A. Licciardello, and O. Puglsi, Appl. Physs. Lett. 51, 907 (1987). 
Figure Captions

Fig. 1 Surface hardness variation of polystyrene bombarded by $350 \mathrm{keV} \mathrm{He}^{+}$and $1 \mathrm{MeV} \mathrm{Ar}^{+}$ions as a function of (a) fluence and (b) dose.

Fig.2 TRIM calculated electronic and nuclear LET as a function of depth for $350 \mathrm{keV}$ He and 1 $\mathrm{MeV}$ Ar for polystyrene.

Fig. 3 Schematic illustration of microstructure evolution for crosslinking and scission dominant regimes. Crosslinking and scission occur simultaneously during irradiation. The ratio of crosslinking to scission depends upon polymer structure but can be altered by using different combinations of ion species, energy, and fluence in HEIB process. Under low LET condition, scissioning type polymers such as PMMA become more solvable at low fluence due to dominant scission but become insoluble at high fluence due to network formation by crosslinking. Under high LET condition, however, scissioning regime can be shifted to crosslinking regime by enhanced radical formation and crosslinking.

Fig. 4 Overlap of two circles for two different radius. The probability of overiap increases with increasing radii for a given distance between the two circle centers.

Fig. 5 Track overlap for circles with radius ratio of 1:2. When circles are laid to cover the same area using an area ratio of 4:1for small and large circles respectively, track overlap occurs earlier for the larger circles as indicated by increasing shade density for single, double, and triple overlap areas.

Fig. 6 Idealized representation of crosslinking regime as a function of dose. At low fluence, ion tracks are isolated and crosslinking occurs at a slower rate in single ion track regime. At intermediate fluence, enhanced crosslinking occurs in regions where tracks overiap. Therefore $\mathrm{G}$ (Single Track) is lower than G(Track Overlap). With increasing LET, effective crosslinking radius increases and track overlap occurs earlier resulting shorter incubation period, but crosslinking rate (slope of the curve) decreases because energy density within the track decreases. Therefore, the total $\mathrm{G}_{t}$ (High LET) is higher than $\mathrm{G}_{\mathrm{t}}$ (Low LET) but the instaneous $\mathrm{G}_{\mathrm{i}}\left(\right.$ High LET) becomes lower than $\mathrm{G}_{\mathrm{i}}$ (Low LET). At high fluence, scission becomes dominant as crosslinking sites are exhausted. The transition from single ion track to track overlap and from track overlap to saturation regime would occur gradually. 

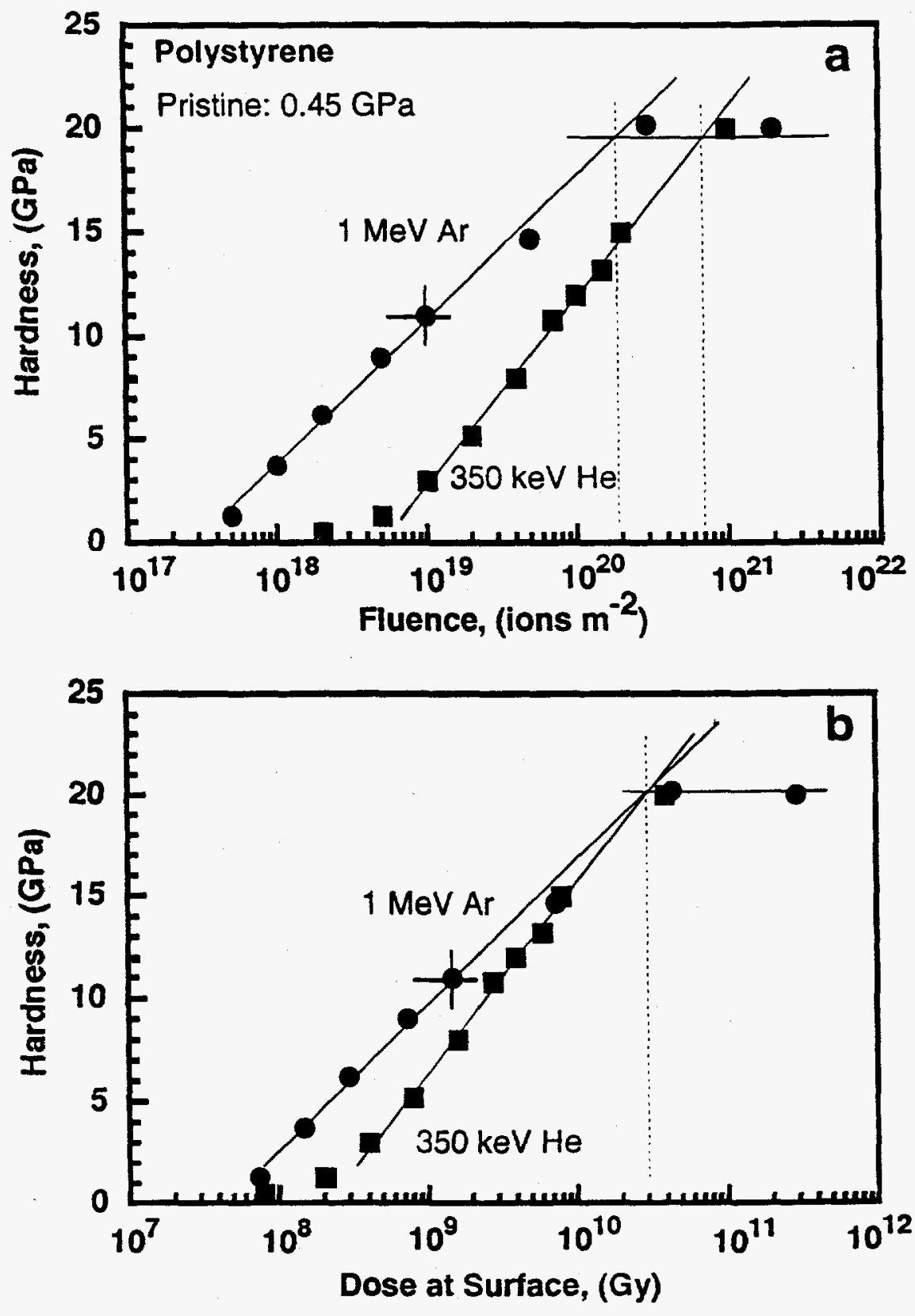

Fig. 1 

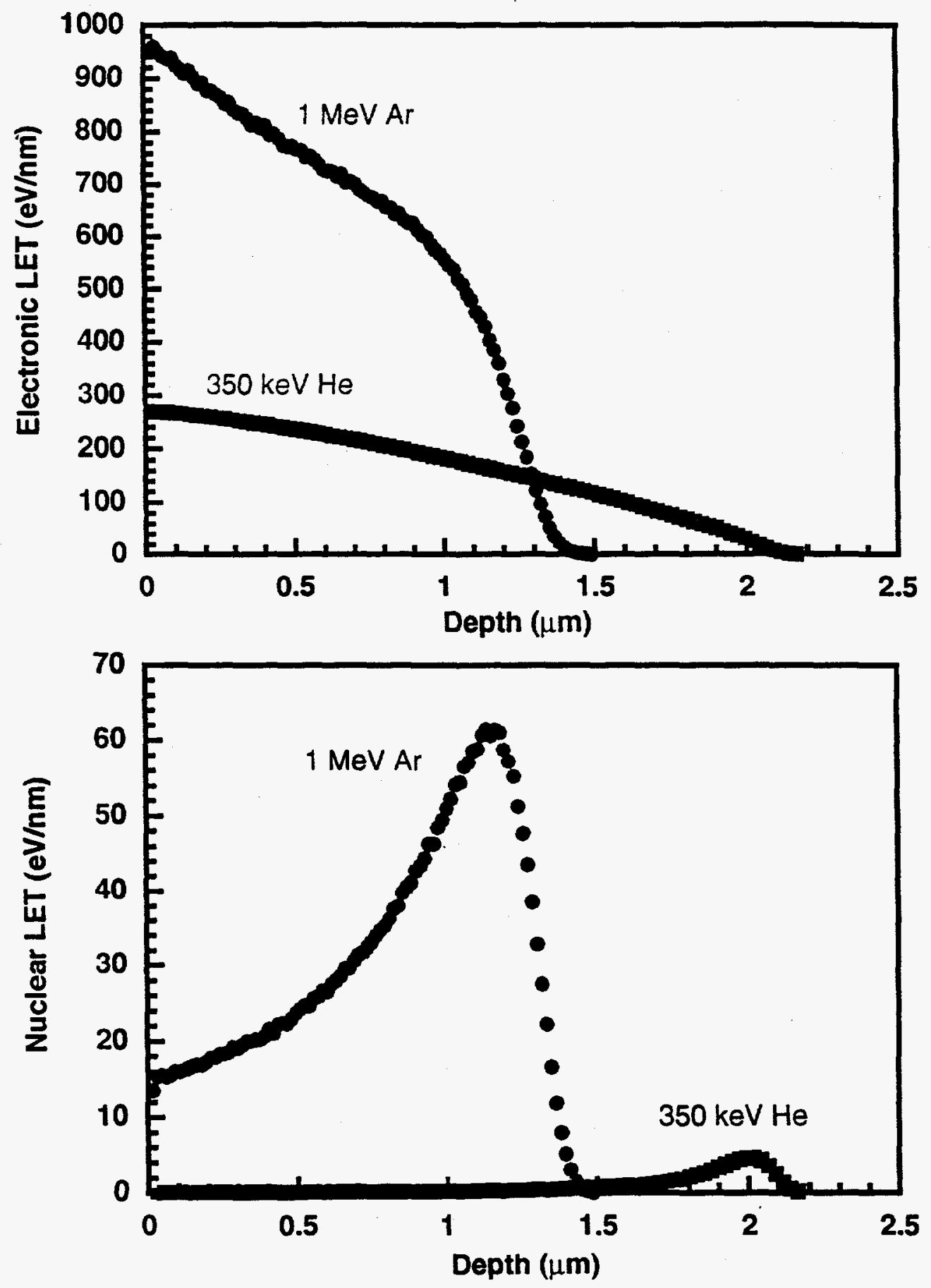

Fig. 2 


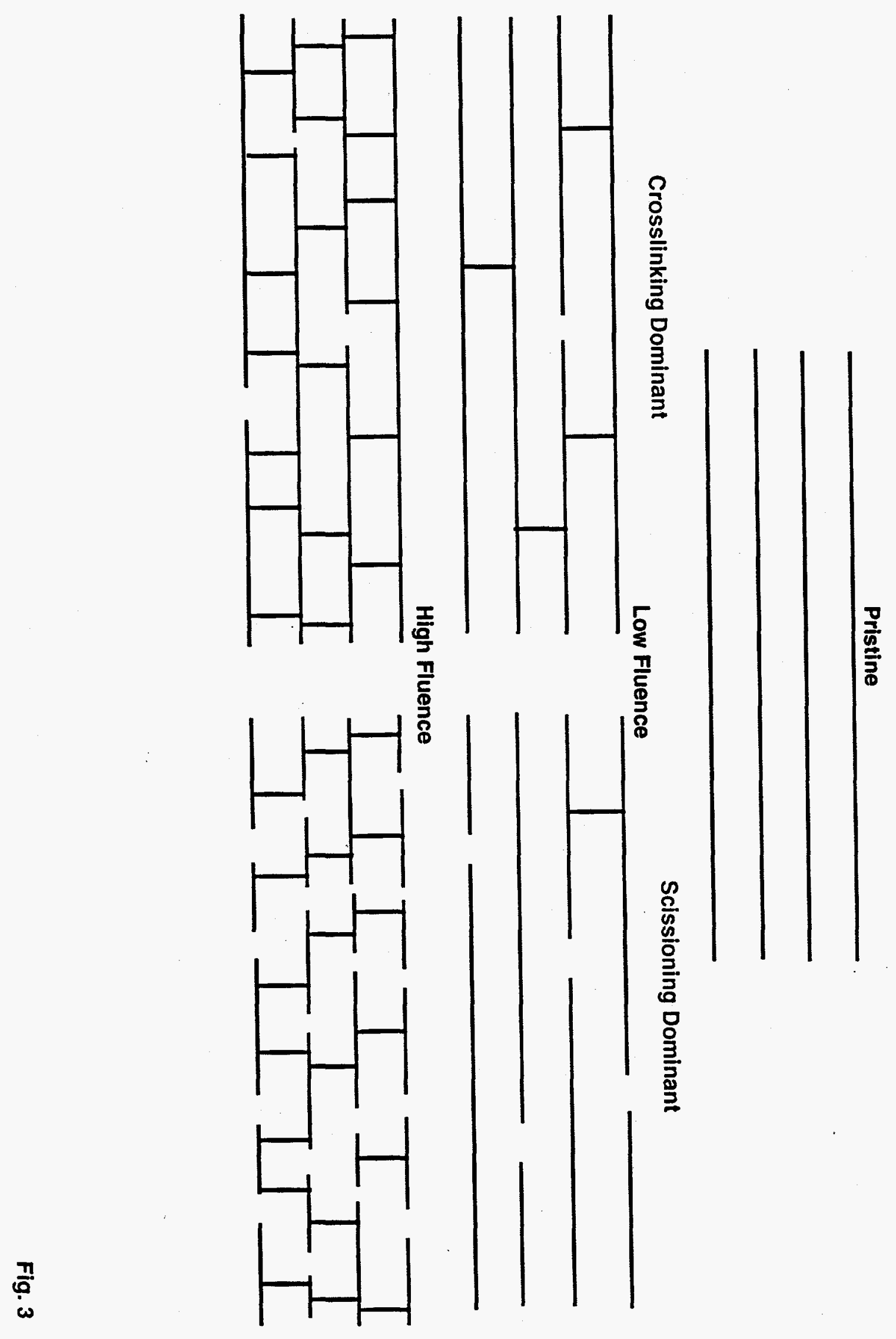




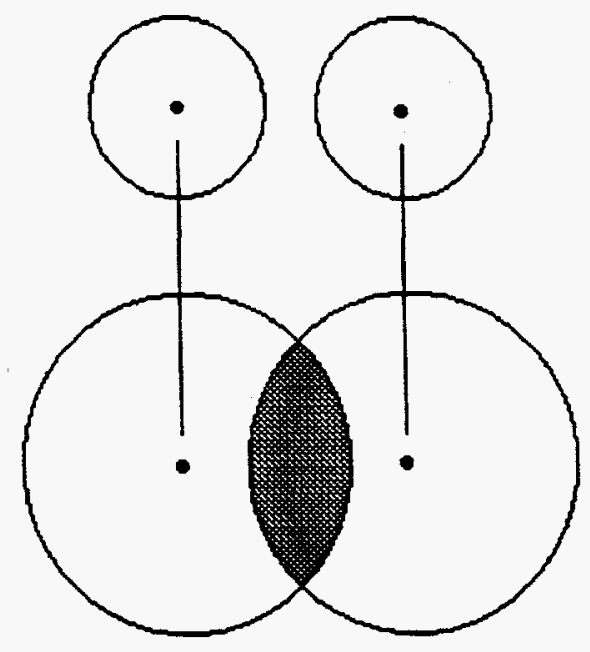

Fig. 4

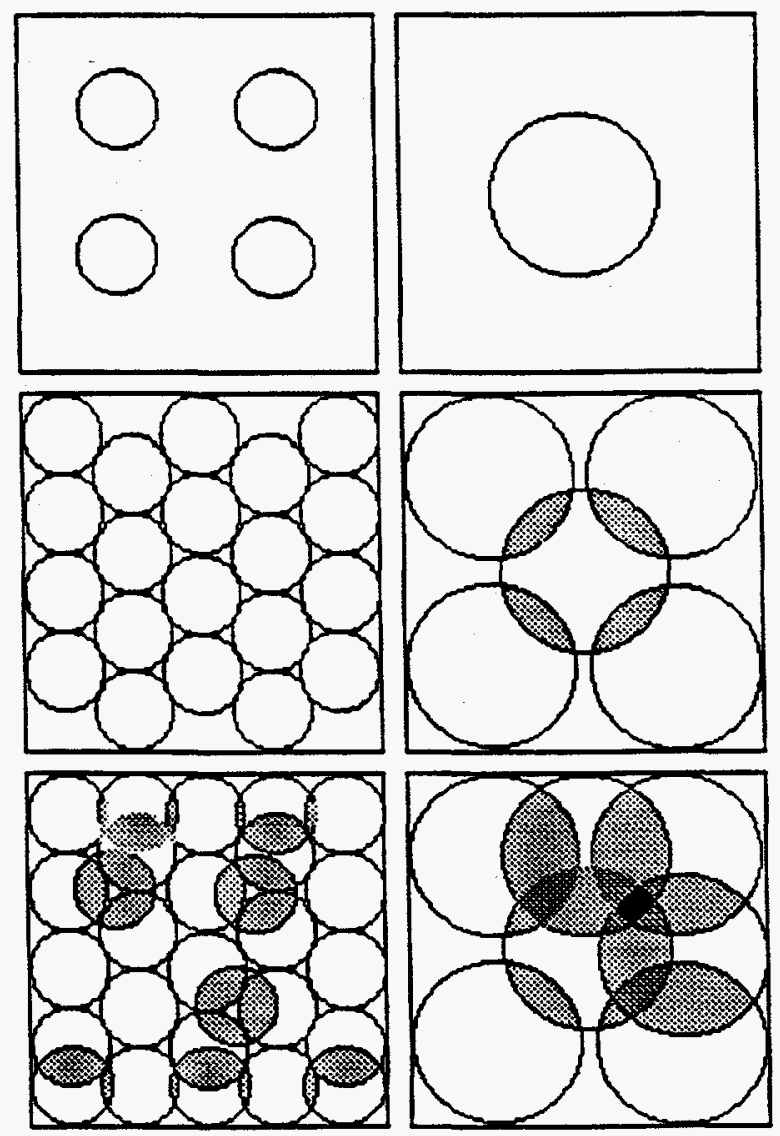

Fig. 5 


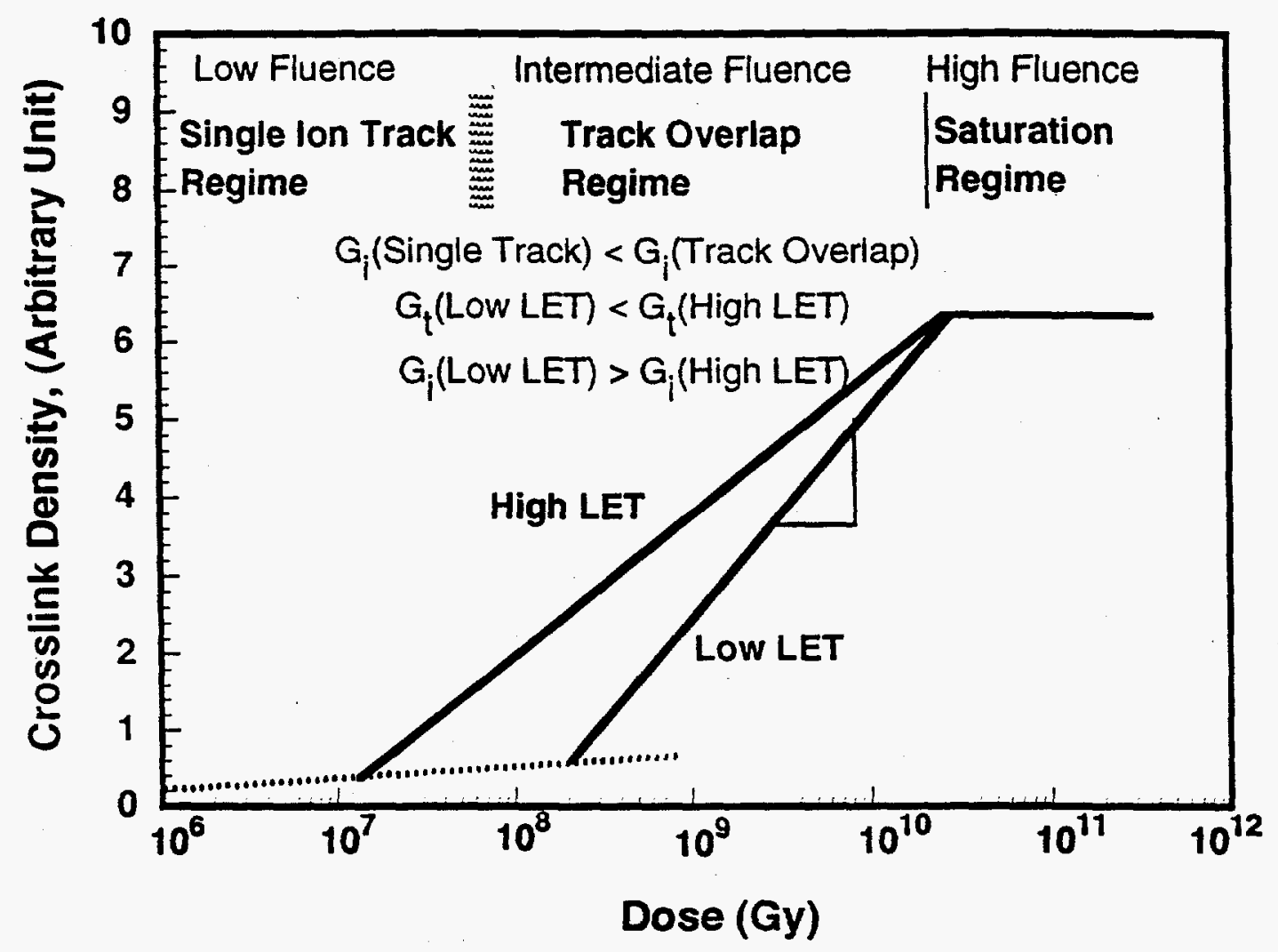

Fig. 6 